\title{
Double-pass versus aberrometric modulation transfer function in green light
}

\author{
Pablo Rodríguez \\ ICMA \\ Consejo Superior de Investigaciones Científicas \\ Universidad de Zaragoza \\ Facultad de Ciencias \\ Plaza San Francisco s/n \\ 50009 Zaragoza, Spain \\ and \\ Universidad de Zaragoza \\ Departamento de Física Aplicada \\ Facultad de Ciencias \\ Plaza San Francisco s/n \\ 50009 Zaragoza, Spain
}

\author{
Rafael Navarro \\ ICMA \\ Consejo Superior de Investigaciones Científicas \\ Universidad de Zaragoza \\ Facultad de Ciencias \\ Plaza San Francisco s/n \\ 50009 Zaragoza, Spain
}

\begin{abstract}
Intraocular scattering can become an important source of optical degradation in the aging eye. To evaluate its relative contribution to the ocular modulation transfer function (MTF), a compact, dual experimental system comprising a laser ray tracing (LRT) wavefront sensor and a double-pass setup is used. An aberrometric MTF is estimated from aberration measurements, whereas a second MTF is derived from the double-pass point-spread function. While the former only accounts for the effect of aberrations (up to seventh order), the double-pass MTF includes the combined effect of both scattering and aberrations. A 532-nm laser light source is used to minimize choroidal scattering. Measurements are done on 19 normal, healthy eyes from three groups of subjects of different ages. The two MTFs are obtained for a 6-mm pupil diameter and partial refractive compensation. Intraocular scattering is modeled as a random wavefront aberration characterized by its variance and correlation length. These parameters are fitted from the differences between both MTFs. Our results show that double-pass and LRT techniques provide similar MTFs for most normal eyes, although small amounts of scattering, or high-order aberrations, could be measured in some eyes. A gradual increase in intraocular scattering with age is also observed. $\odot 2007$ Society of PhotoOptical Instrumentation Engineers. [DOI: 10.1117/1.2756539]
\end{abstract}

Keywords: vision; aberrations; scattering; modulation transfer function; glare.

Paper 06340R received Nov. 20, 2006; revised manuscript received Mar. 29, 2007; accepted for publication May 4, 2007; published online Jul. 19, 2007.

\section{Introduction}

The optical quality of the eye is determined by three factors: diffraction, aberrations, and scattering. Diffraction imposes a fundamental limit to the performance of any optical system, whereas aberrations constitute the main source of optical degradation in normal eyes. Several methods have been developed or adapted in the last decade to cope with the special conditions of in-vivo objective measurements of human eye aberrations (i.e., light passing twice through the optical media): the Hartmann-Shack (HS) wavefront sensor, ${ }^{1}$ the laser ray tracing (LRT) method, ${ }^{2}$ the crossed-cylinder aberroscope, ${ }^{3}$ and the spatially resolved refractometer, ${ }^{4}$ among others. Commercial versions of these systems have favored an increasing number of studies on eye monochromatic aberrations, while their clinical application has quickened their development, especially in the case of refractive surgery. Typically, the ocular wavefront aberration is expanded in a set of Zernike polynomials up to a certain order ${ }^{5}$ (usually from fifth to seventh). This means that finer details of the wavefront aberration, typically below $100 \mu \mathrm{m}$, are not recovered by these devices. Therefore, by measuring the coarse features of the wavefront aberration, aberrometry focuses on small-angle effects. These mainly affect the core of the point-spread function, and hence

Address all correspondence to Rafael Navarro, ICMA, Facultad de Ciencias, Plaza San Francisco s/n, 50009 Zaragoza, Spain. Tel: (34) 976 762782; Fax: (34) 976 761233; E-mail: rafaelnb@unizar.es neglect the wide-angle effects, dominated by scattering (and very high-order aberrations).

Concerning intraocular scattering, its objective measurement has experienced a much slower development, despite its role in such important pathologies as cataracts or dry eye. In the medical practice, detection of intraocular scattering has relied traditionally on backscattering measurements (slit lamp, Scheimpflug cameras, etc.). Recently, this field has been widened by the application of dynamic light scattering, which provides the size distribution of possible scatterers in the human lens. ${ }^{6}$ Forward scattering measurement methods were first developed to identify the sources of glare in the human eye. Subjective veiling-glare functions were first obtained many decades ago, ${ }^{7-11}$ and several objective in-vitro measurements of scattering in the eye $\mathrm{e}^{12}$ or in the crystalline lens ${ }^{13}$ have been reported as well. Decades of studies on disability glare have led to the recent publication of the CIE equations for disability glare, ${ }^{14}$ which establish a set of glare equations valid over several angle domains.

However, objective in-vivo forward scattering measurement techniques have not been available until recently. Basically, the methods described in the literature rely on doublepass imaging techniques, which try to quantify the optical effects of scattering on the retinal image. Double-pass imaging has been employed either alone, or in combination with other measurements, to provide several metrics of the degree

1083-3668/2007/12(4)/044018/9/\$25.00 @ 2007 SPIE 


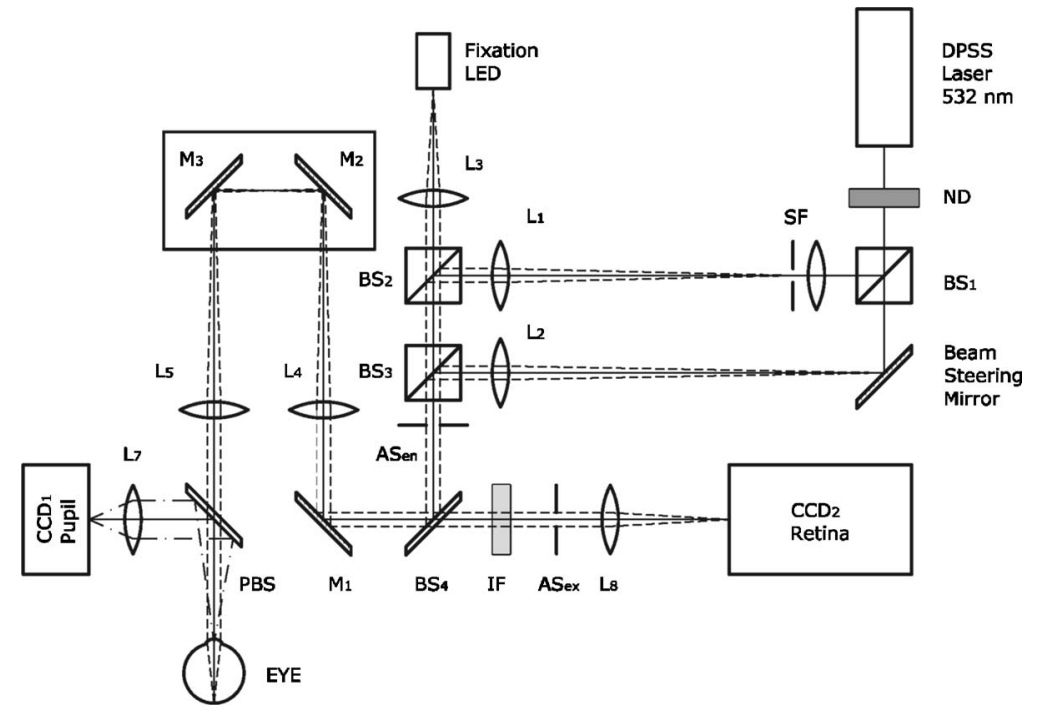

Fig. 1 An schematic view of the dual optical setup. ND, neutral density filter; BS, beamsplitter; SF, spatial filter; $\mathrm{AS}_{\mathrm{en}}$, aperture stop (entrance); $\mathrm{AS}_{\mathrm{ex}}$ aperture stop (exit); $M$, mirror; PBS, pellicle beamsplitter; CCD, charge-coupled device; IF, interference filter; $L_{1}, 120-\mathrm{mm}_{\text {focal lens; }} L_{2}, 140 \mathrm{~mm}$; $\mathrm{L}_{3}, 80 \mathrm{~mm} ; \mathrm{L}_{4}, \mathrm{~L}_{5}$, and $\mathrm{L}_{8}, 100 \mathrm{~mm}$; and $\mathrm{L}_{7}, 25 \mathrm{~mm}$.

of scattering, ranging from the spreading of the double-pass image $^{15-17}$ to the degree of polarization. ${ }^{18}$ There are two main limitations in these objective double-pass imaging approaches to analyze scattering. Intraocular scattering produces a wide and dim halo on the retina. This wide spread derives from particles of small size, a few micrometers, and the intensity is typically several orders of magnitude lower than the peak of the point-spread function (PSF). These two properties make the detection of scattering halos particularly difficult by double-pass systems, since the field of view in a double-pass image is usually narrow, 2 to $4 \mathrm{deg}$, and the dynamic range of the charge-coupled device (CCD) cameras is limited to about three orders of magnitude. Furthermore, depending of the wavelength used (i.e., red or near-infrared), the contribution of light scattered by blood vessels from the choroids to the double-pass image may be even stronger than the faint retinal halo, thus masking it. Consequently, we have designed carefully our experimental system to optimize these variables, namely field of view, dynamic range, and wavelength.

In addition to a careful experimental design, a further enhancement of these methods can be achieved by making use of an adequate theoretical framework to improve the data analysis. Several models of intraocular scattering have been proposed in the literature, ${ }^{19-24}$ either based on theoretical or empirical backgrounds. Nevertheless, we have opted for a model based on the concept of equivalent diffuser, ${ }^{25}$ i.e., a phase screen or rough surface. This is a simple approach that permits us to apply a rigorous theoretical framework.

Our experimental approach is similar to those developed in Refs. 26 and 27, and hence it is based on a combination of both aberrometric and double-pass measurements. A laser ray tracing aberrometer ${ }^{2}$ was used to obtain the wavefront aberration (up to a certain order, limited by the finite spatial sampling) and information on the Stiles-Crawford effect. From these data, the aberrometric MTF was then calculated. A double-pass device ${ }^{28}$ provided the complete (that is, including all aberrations and scattering) modulation transfer function of the eye. An estimate of the degrading effect of both scattering and higher-order aberrations could be obtained from a direct comparison of both MTF measurements, while a simple, rough surface equivalent diffuser model of intraocular scattering $^{25}$ permitted the quantification of the degree of scattering in a straight manner. The comparison between MTFs is simplified by the fact that both apparatus are built into a single experimental system, thus allowing a careful correction of possible differences in focus or in eye alignment, which could yield misleading results. Another fundamental advantage of our experimental setup is the use of a green laser source $(532 \mathrm{~nm})$, which minimizes the scattering from blood vessels and deeper layers other than the photoreceptor outer segments. ${ }^{29}$ This additional source of scattering could have a greater influence on double-pass aerial images obtained under red or infrared illumination. ${ }^{30}$ Our results show that, in most subjects, the differences between aberrometric and doublepass MTFs are small and below measurement errors. As in previous studies, ${ }^{17,31-33}$ we also observe a trend to increase intraocular scattering with age.

\section{Materials and Methods}

\subsection{Apparatus}

A laser ray tracing wavefront sensor and a double-pass setup were combined into a compact dual experimental system (Fig. 1) to obtain the ocular MTF in two different ways. Both subsystems share most of the optical path, including a Badal optometer and a cooled scientific camera $\left(\mathrm{CCD}_{2}\right)$ to register both LRT and double-pass retinal images. An additional camera $\left(\mathrm{CCD}_{1}\right)$ was used to monitor the pupil. This permits us both a better pupil alignment and later the chance to correct those small pupil displacements that may occur during aberration measurements. A green laser source $(532 \mathrm{~nm})$ was used to minimize the contribution of scattering ${ }^{30}$ from blood vessels (retina and choroid). The laser ray tracing method has been described in detail before. ${ }^{2}$ This particular implementa- 
tion incorporates a single two-axis tip/tilt beam steering mirror. In this way, we avoid the astigmatism inherent in twomirror scanners. The system delivers, sequentially, a bundle of laser pencils (rays) that samples the pupil in a hexagonal grid. Although in the present experiment we used 37 rays, both the sampling pattern and density are freely programmable. For each ray, two images corresponding to the pupil and the retina are recorded simultaneously, the former to detect any possible eye movement. In the current configuration, each measurement takes $2 \mathrm{sec}$, closely matching typical double-pass exposures. The maximum laser power on the cornea was $16 \mu \mathrm{W}$, well below international safety standards. ${ }^{34}$ An expansion in Zernike polynomials (up to seventh order) of the wavefront aberration was obtained from the centroids of the recorded retinal spots. The integrated intensity of each spot was then used to obtain an estimate of the Stiles-Crawford effect, which also affects the MTF for the pupil diameters involved (6 mm). From these data, we built the complex pupil function, ${ }^{35}$ and computed the modulus of its autocorrelation, that is, the aberrometric MTF. This aberrometric MTF does not include the effect of scattering or very high order of aberrations, since details of the wavefront aberration finer than the spacing between rays (about $800 \mu \mathrm{m}$ here) are missed. In addition, there is a lower limit set by the spatial average of each Gaussian ray in the pupil plane (roughly $150 \mu \mathrm{m}$ fullwidth at half-maximum). These missed details will have an effect mainly on the lower frequencies in the MTF.

The second subsystem, sharing the same optical path as the LRT, is a symmetric double-pass device. A spatially filtered collimated beam of light is delivered into the eye and the aerial image is recorded. Our setup (see Fig. 1) uses a spatial filter (SF) consisting of a $40 \times$ microscope objective and a $10-\mu \mathrm{m}$ pinhole in its focus; a $120-\mathrm{mm}$ focal lens $\left(\mathrm{L}_{1}\right)$ collimates the point-like source, and two artificial pupils of $6 \mathrm{~mm}$ diam $\left(\mathrm{AS}_{\mathrm{en}}\right.$ and $\left.\mathrm{AS}_{\mathrm{ex}}\right)$ guarantee a symmetric configuration. The camera $\left(\mathrm{CCD}_{2}\right)$ uses a $100-\mathrm{mm}$ objective covering a total field angle of $3.9 \times 3.9 \mathrm{deg}$. A small correction to account for the finite sampling of the camera was applied. Exposure time never exceeded $5 \mathrm{sec}$ (typically $2.5 \mathrm{sec}$ ), and the incident power at the cornea was below $0.5 \mu \mathrm{W}$, again observing international safety standards. When both entrance and exit pupils are equal, and under the assumption of isoplanatism and incoherent imaging (long exposures), the aerial image corresponds to the autocorrelation of the onepass point-spread function (PSF). ${ }^{28}$ Thus, using the WienerKhinchin theorem, the double-pass MTF can be calculated as the square root of the inverse Fourier transform of the doublepass image. The symmetric double-pass system does not permit us to retrieve the phase transfer function (PTF), and hence the complex optical transfer function (OTF), but only the MTF is necessary to determine the contribution of scattering to the optical quality of the eye.

Aberrations have a deleterious effect on the MTF, especially for bigger pupils, and hence they must be either minimized or balanced, equally in both subsystems. Since most of the optical path is common to both subsystems, any discrepancy would come from the collimating lenses $\left(\mathrm{L}_{1}\right.$ and $\mathrm{L}_{2}$ in Fig. 1). Additionally, the CCD objective must be placed to ensure a symmetric configuration in the double-pass setup. This implies that both the pinhole (double pass) and the steer- ing mirror (LRT) should be equally imaged on the CCD plane with the highest possible precision. With this aim, we performed an iterative calibration by means of a set of trial lenses placed in front of an artificial eye consisting of a 200-mm lens doublet corrected for spherical aberration and a rotating white screen that acts as an "artificial retina." This iterative calibration permitted us a fine tuning of the position of the collimating lenses, $\mathrm{L}_{1}$ and $\mathrm{L}_{2}$, and CCD objective $\mathrm{L}_{8}$. Finally, a finer calibration was performed using a set of phase plates, placed in front of the artificial eye, which generates known amounts of single mode aberrations. ${ }^{36}$

\subsection{Subjects}

Measurements were done on 19 normal, healthy eyes from three groups of subjects of different ages $(28 \pm 3,42 \pm 3$, and $65 \pm 2$ years). A partial compensation of spherical ametropia was applied by means of a Badal optometer, according to previous aberration measurements. Subjects' pupils were dilated by instillation of 2 drops of tropicamide $1 \%$; the second drop, 5 min after the first one. The measurements started $30 \mathrm{~min}$ after the first drop. Each session (typically including from five to ten consecutive measurements with each subsystem) lasted about half an hour per eye. Additional drops of tropicamide were instilled if the session extended beyond this point. The two MTFs were obtained for a 6-mm pupil diameter. All the subjects underwent a previous slit-lamp examination where early stage cataracts (grade 1 or less, according to the Lens Opacities Classification System III $^{37}$ ) were detected in six eyes from the two older groups. These eyes are subsequently identified throughout the study. Informed consent was obtained from all the subjects, and the experimental procedures followed the tenets of the Declaration of Helsinki.

Laser ray tracing measurements and double-pass images were processed after subtraction of background images to reduce the amount of stray light from the optical setup. Additionally, double-pass images were thresholded to eliminate readout noise from the camera and possible background and stray light coming from specular reflections from the cornea or other optical surfaces of the system. For this purpose, a small patch (16 min arc) in the corners of the double-pass image, about $2.6 \mathrm{deg}$ away from the center, was analyzed. The threshold level was calculated as the mean value plus three standard deviations. This threshold was subsequently taken into account when obtaining the aberrometric MTF. Hence, we first calculated the double-pass PSF from the aberrometric data and then applied the dynamic range of the camera (maximum and threshold intensities) before computing the MTF, otherwise some of the intensity in the tails of the aberrometric point-spread function would be included in the calculation (after neglecting it in the double-pass measurements). Nevertheless, this correction was usually negligible, except for highly aberrated eyes.

\subsection{Scattering Model}

Intraocular scattering was modeled as an equivalent phase screen Gaussian diffuser placed at the pupil plane. ${ }^{25}$ This kind of phase screen can be described as a random wavefront aberration with Gaussian distribution and zero mean. Its scattering properties are then characterized by the standard deviation $\sigma$ (roughness), and the correlation length $r_{0}$ (grain size) of the 


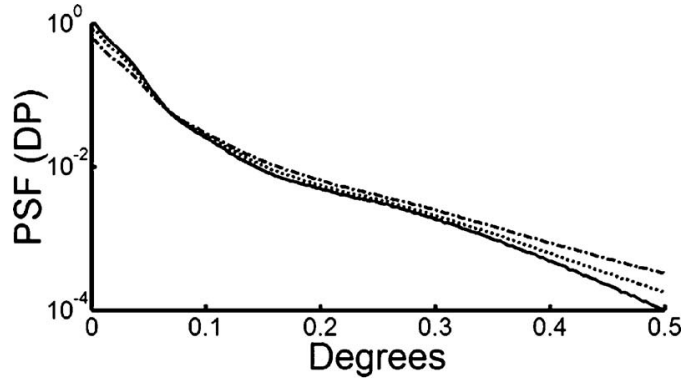

(a)

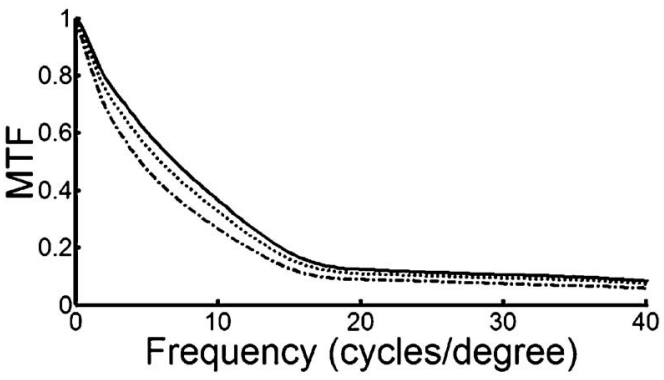

(b)

Fig. 2 Radial average of (a) the double-pass point spread functions and (b) modulation transfer functions corresponding to a 44-years-old subject's aberrations (solid line) plus equivalent diffusers with the same correlation length, $r_{0}=100 \mu \mathrm{m}$, but different RMS roughness, $\sigma=0.030 \mu \mathrm{m}$ (dotted line) and $\sigma=0.050 \mu \mathrm{m}$ (dot-dashed line).

optical path difference (OPD) introduced by the diffuser. Phase difference is directly proportional to the OPD, both quantities being related through the wavelength. An exponential autocorrelation function of the OPD was employed, since it produces a theoretical halo with an angular dependence proportional to $\theta^{-3}$, similar to that of the CIE small angle disability glare equation ${ }^{14}$ at small angles. Thus, the complete wavefront aberration of the eye can be decomposed into two additive terms: a deterministic term $\left(W_{a b}\right)$ and a random component $\left(W_{\text {scat }}\right)$. Therefore, the complete optical transfer function (OTF) can be expressed as the autocorrelation of the complex pupil function:

$$
\begin{aligned}
\operatorname{OTF}_{\mathrm{ab}+\mathrm{sc}}\left(\frac{\xi}{\lambda f^{\prime}}, \frac{\eta}{\lambda f^{\prime}}\right)= & \int_{-\infty}^{+\infty} \int_{-\infty}^{+\infty} P\left(\xi^{\prime}, \eta^{\prime}\right) P^{*}\left(\xi^{\prime}-\xi, \eta^{\prime}-\eta\right) \\
& \times \exp \left\{i k \left[W_{\text {scat }}\left(\xi^{\prime}, \eta^{\prime}\right)\right.\right. \\
& \left.\left.-W_{\text {scat }}\left(\xi^{\prime}-\xi, \eta^{\prime}-\eta\right)\right]\right\} d \xi^{\prime} d \eta^{\prime}
\end{aligned}
$$

where $\xi$ and $\eta$ are pupil coordinates, $\lambda$ is the wavelength, $f^{\prime}$ is the focal length, $k$ is the wave number, and $P(\xi, \eta)$ $=T(\xi, \eta) \exp \left[i k W_{a b}(\xi, \eta)\right]$ is the complex pupil function, where $T(\xi, \eta)$ is the effective pupil transmittance. Assuming that $W_{a b}$ and $W_{\text {scat }}$ are uncorrelated, their effect on the OTF can be separated into two multiplicative terms,

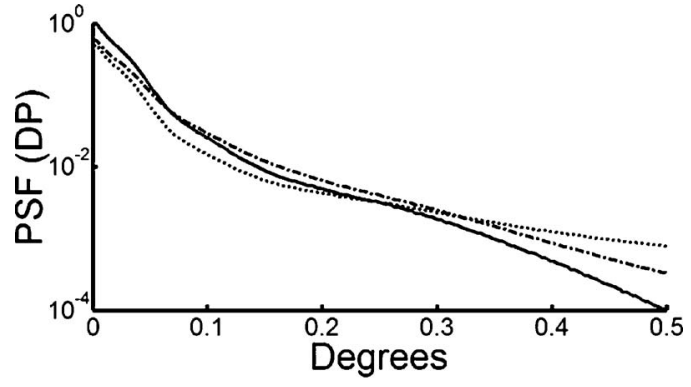

(a)

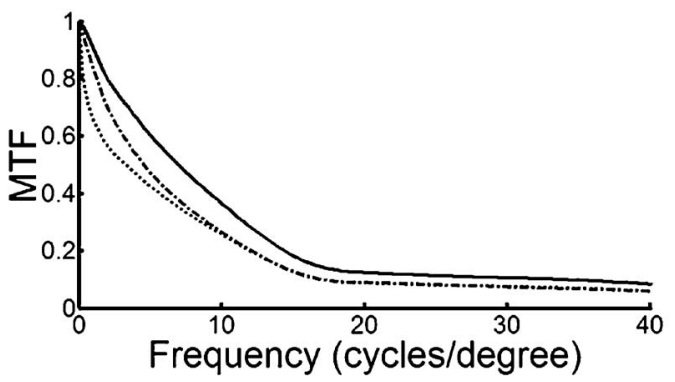

(b)

Fig. 3 Radial average of (a) the double-pass point spread functions and (b) modulation transfer functions corresponding to a 44-years-old subject's aberrations (solid line) plus equivalent diffusers with the same RMS roughness, $\sigma=0.050 \mu \mathrm{m}$, but different correlation length, $r_{0}=10 \mu \mathrm{m}$ (dotted line) and $r_{0}=100 \mu \mathrm{m}$ (dot-dashed line).

$$
\begin{aligned}
\mathrm{OTF}_{\mathrm{ab}+\mathrm{sc}}\left(\frac{\xi}{\lambda f^{\prime}}, \frac{\eta}{\lambda f^{\prime}}\right)= & \mathrm{OTF}_{\mathrm{ab}}\left(\frac{\xi}{\lambda f^{\prime}}, \frac{\eta}{\lambda f^{\prime}}\right) \\
& \times \int_{-\infty}^{+\infty} \int_{-\infty}^{+\infty} \exp \left\{i k \left[W_{\text {scat }}\left(\xi^{\prime}, \eta^{\prime}\right)\right.\right. \\
& \left.\left.-W_{\text {scat }}\left(\xi^{\prime}-\xi, \eta^{\prime}-\eta\right)\right]\right\} d \xi^{\prime} d \eta^{\prime}
\end{aligned}
$$

Following the formulation developed by Beckmann, ${ }^{38,39}$ we can average then over an ensemble of random realizations, and obtain the MTF:

$$
\begin{aligned}
\operatorname{MTF}_{\mathrm{ab}+\mathrm{sc}}(u, v)= & \operatorname{MTF}_{\mathrm{ab}}(u, v) \\
& \times \exp \left\{-k^{2} \sigma^{2}\left[1-C\left(\lambda f^{\prime} u, \lambda f^{\prime} v\right)\right]\right\},
\end{aligned}
$$

where $u$ and $v$ are frequency coordinates, and $\sigma$ and $C\left(\lambda f^{\prime} u, \lambda f^{\prime} v\right)$ are the standard deviation and the autocorrelation function of $W_{\text {scat }}$, respectively. As we said before, we assume that the autocorrelation is an exponential function $C(u, v)=\exp \left[-\left(u^{2}+v^{2}\right)^{1 / 2} / r_{0}\right]$. Finally, the exponential in Eq. (3) can be expanded in the following Taylor series:

$$
\begin{aligned}
\operatorname{MTF}_{\mathrm{ab}+\mathrm{sc}}(u, v)= & \operatorname{MTF}_{\mathrm{ab}}(u, v) \times \exp \left(-k^{2} \sigma^{2}\right) \\
& \times \sum_{n=0}^{\infty} \frac{k^{2 n} \sigma^{2 n} C^{n}\left(\lambda f^{\prime} u, \lambda f^{\prime} v\right)}{n !} .
\end{aligned}
$$

The number of terms to preserve in the series of Eq. (4) is related to the magnitude of $k^{2} \sigma^{2}$. Since in normal healthy eyes the amount of scattering is known to be small, we could assume that the weak diffuser approximation $\left(k^{2} \sigma^{2} \ll 1\right)$ may be 


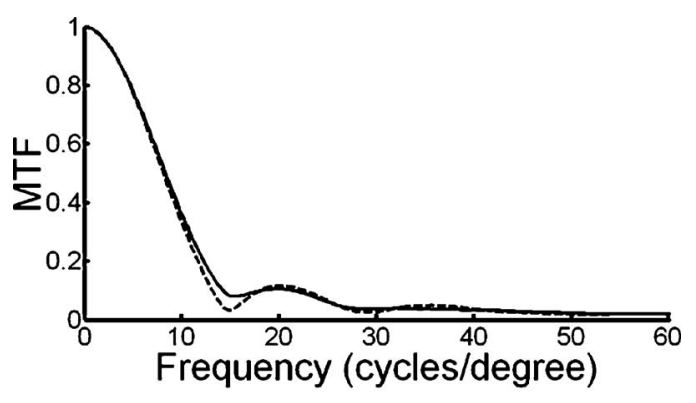

(a)

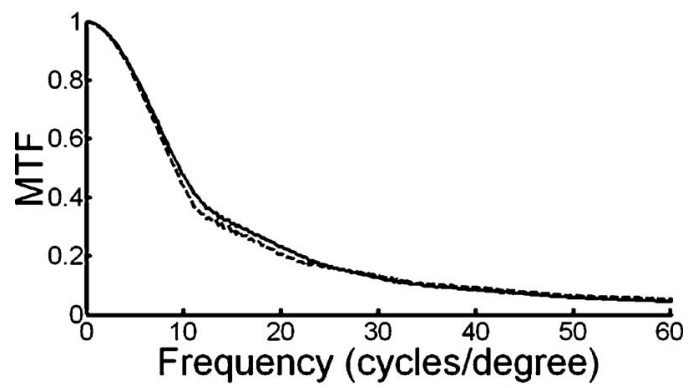

(b)

Fig. 4 Calibration results for second-order aberrations, (a) defocus $(0.25 \mathrm{D})$ and (b) astigmatism (-0.25 at $90 \mathrm{deg})$, displaying the radial average of the aberrometric (dashed line) and double-pass (solid line) modulation transfer functions.

valid. In such a case, we can neglect the contribution of terms with $n>1$ in Eq. (4):

$$
\begin{aligned}
\operatorname{MTF}_{\mathrm{ab}+\mathrm{sc}}(u, v)= & \mathrm{MTF}_{\mathrm{ab}}(u, v) \times \exp \left(-k^{2} \sigma^{2}\right) \\
& \times\left\{1+k^{2} \sigma^{2} \exp \left[-\frac{\lambda f^{\prime}}{r_{0}}\left(u^{2}+v^{2}\right)^{1 / 2}\right]\right\} .
\end{aligned}
$$

For young healthy subjects, this weak diffuser approximation may be good enough. Nevertheless, to be more general, typically we used up to five terms $(n=4)$ in Eq. (4), trying to balance accuracy and computational simplicity.

The theoretical effect of the parameters $\sigma$ and $r_{0}$ on the double-pass PSF and MTF is illustrated with two examples in Figs. 2 and 3. The standard deviation (or RMS roughness) $\sigma$ is straightforwardly related to the quotient of MTF volumes and thus to the quotient of Strehl ratios. As can be seen in Fig. 2(a), as the roughness increases, the amount of light in the core of the PSF diminishes while it grows in the tails. In other words, $\sigma$ determines the amount of scattered light that goes to the halo. As the halo grows, there is a predominance of low spatial frequencies in the PSF and, consequently, a decrease of the MTF at medium to high frequencies. This translates into a reduction of the volume in the MTF [Fig. 2(b)]. On the other hand, the parameter $r_{0}$ is linked to the spatial extent of the scattering halo: the smaller the correlation length, the wider the halo and vice versa [Fig. 3(a)]. Thus, a small correlation length would have a strong impact on low frequencies, whereas a higher correlation length would affect a higher range of frequencies [Fig. 3(b)]. This behavior can be easily predicted from Eq. (5). In fact, aberrations such as coma or

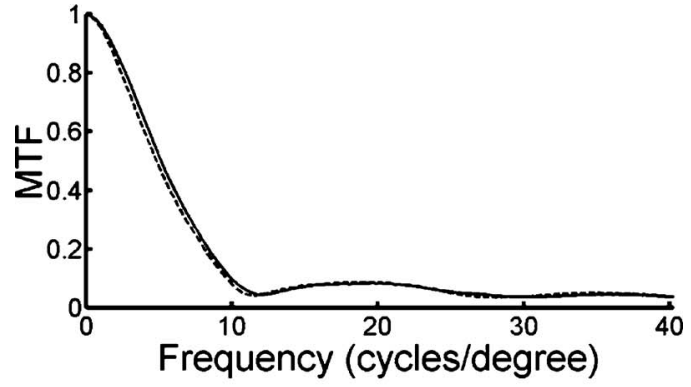

(a)

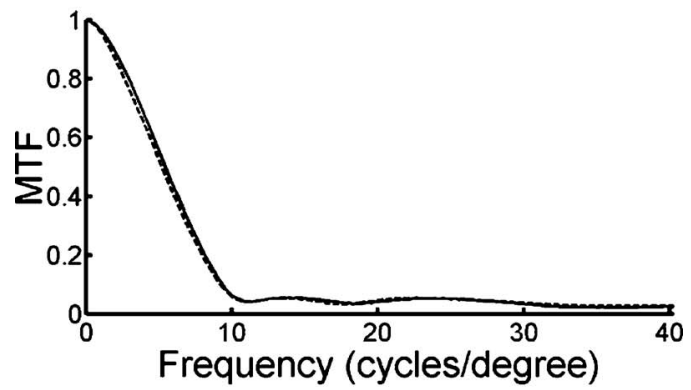

(b)

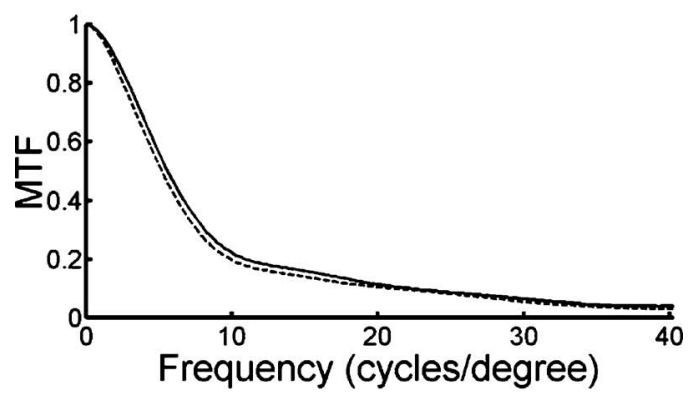

(c)

Fig. 5 Radial average of the double-pass (solid line) and aberrometric (dashed line) modulation transfer functions for some samples of a calibration set of monomode phase plates. From top to bottom, results corresponding to plates (a) $Z_{4}^{-2}(0.333 \mu \mathrm{m})$, (b) $Z_{6}^{2}(0.233 \mu \mathrm{m})$, and (c) $Z_{7}^{5}(0.221 \mu \mathrm{m})$.

spherical aberration can be seen as realizations of a random wavefront aberration with a very high correlation length (of the order of the pupil radius).

The theoretical formulation of the model [Eq. (4)] was used to perform a nonlinear fit to obtain both the standard deviation $\sigma$ and the correlation length $r_{0}$ of the equivalent phase screen diffuser, from the measured 2-D MTFs (that is, $M T F_{a b}$ and $\left.M T F_{a b+s c}\right)$. In this analysis, only values of the MTFs up to an effective cut-off frequency were considered. This cut-off frequency was defined as the angular frequency where the radially averaged MTF arrive at a lower plateau (constant experimental noise level). Initial guess values for the standard deviation were estimated from the quotient between the double-pass and the aberrometric MTFs [first term in the series of Eq. (4)]. For the correlation length $r_{0}$, we used a constant initial guess of $7 \mu \mathrm{m}$, roughly corresponding to the wavefront sampling equivalent to the double-pass image width. In addition, $7 \mu \mathrm{m}$ is compatible with the size of potential scatterers such as epithelium cells in the lens. Convergence was usually reached after a few iterations. 


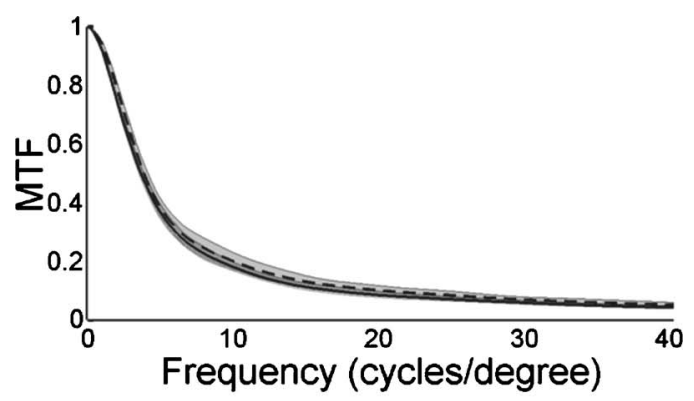

(a)

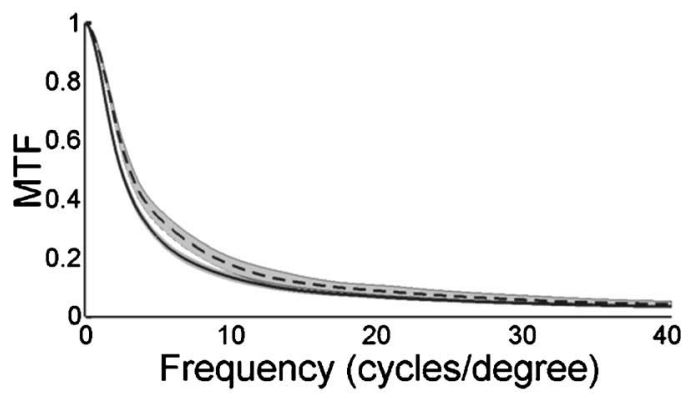

(b)

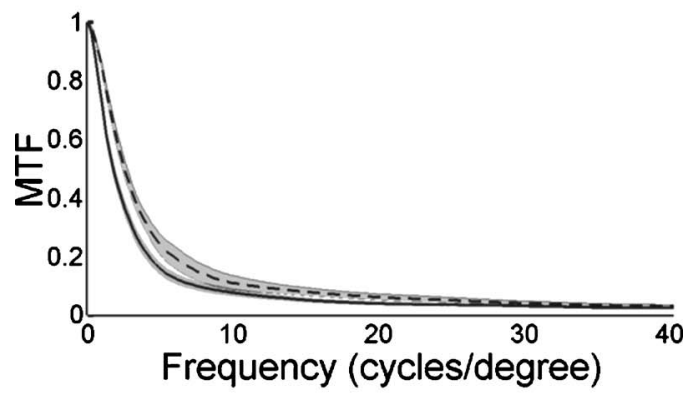

(c)

Fig. 6 Radial average of the double-pass (solid line) and aberrometric (dashed line) modulation transfer functions averaged over each age group. Also displayed are the confidence intervals (shadowed areas). From top to bottom, results correspond to subjects in the (a) young, (b) middle-aged, and (c) oldest groups.

\section{Results}

The results of the calibration on the artificial eye are displayed in Figs. 4 and 5. Figure 4 compares the radial average (computed as the mean over all orientations) of the double-pass (continuous lines) and aberrometric (dashed lines) MTFs of pure defocus [Fig. 4(a)] and pure astigmatism [Fig. 4(b)]. Figure 5 shows a similar comparison, now for three different higher-order Zernike modes $\left(Z_{4}^{-2}, Z_{6}^{2}, Z_{7}^{5}\right)$ produced by the corresponding three phase plates from the calibration set. In all cases, both MTFs are almost overlapped, which means that either scattering or aberrations of higher orders were not detected by our device. This behavior was expected in all cases represented in both figures due to the high optical quality of the artificial eye lens as well as the photoresist layer and glass substrate in the case of the phase plates. Nevertheless, such a remarkable agreement was reached only after a careful and methodical calibration of the dual system.

In Fig. 6 we summarize the MTF measurements in human eyes. Each panel displays the mean aberrometric (dashed line)

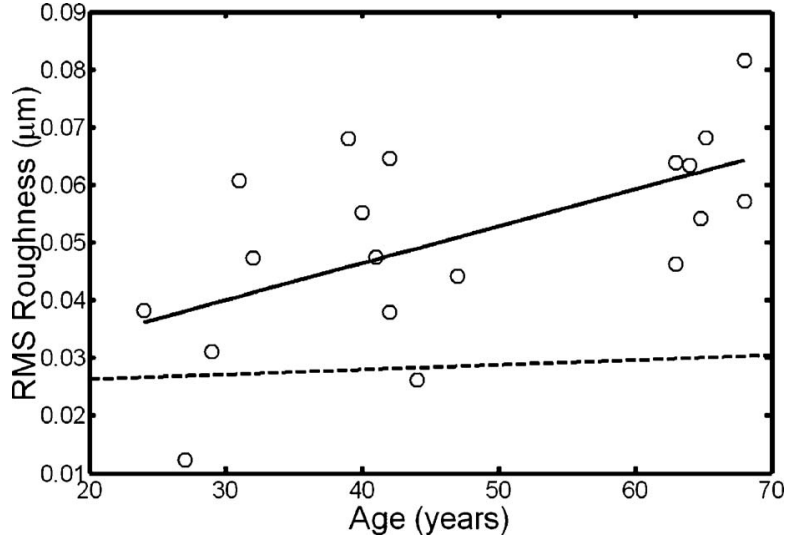

Fig. 7 Root-mean-square (RMS) roughness of the equivalent diffuser versus age (all subjects). The continuous line corresponds to the linear regression ( $r=0.503, p=0.028)$. The dashed line shows the roughness expected from the CIE small angle disability glare equation ( $\sigma$ $=0.027 \mu \mathrm{m})$.

and double-pass (continuous line) MTFs (radial average), together with their standard deviations (shaded areas), for each of the three age groups. In our population of human eyes, the double-pass device always provided a lower MTF, meaning that there were either some very high-order residual aberrations or some scattering not detected by the aberrometer, but affecting the double-pass MTF. It must be noted that for most subjects (11 out of 19), the measurement error in both devices is of the same order of magnitude as the difference between the aberrometric and double-pass mean values. If we perform a t-test on the corresponding Strehl ratios (that is, the volumes under the bidimensional MTFs), we find that for these 11 eyes, the difference is not statistically significant $(p>0.05)$. However, in the oldest group [Fig. 6(c)], most eyes presented a clear difference between the aberrometric and double-pass Strehl ratios ( $p<0.05$ for five out of seven eyes). Therefore, in this group of subjects, an aberrometer would not provide a complete measure of the ocular optical quality, but a biased measure somewhat higher, and hence it would overestimate the real optical performance. If we compare the three panels of Fig. 6, there is a clear trend of the two MTFs to decline with age, as expected. ${ }^{40}$ In addition, both MTFs tend to separate, which suggests a changing balance between coarse and fine details of the wavefront with age. In other words, aberrations seem to increase with age, but scattering (and very highorder aberrations) increases more. Experimental errors limit the sensitivity of this method so that differences are significant only in older eyes, but the increasing relative contribution of scattering becomes patent in these average MTFs.

Our analysis of the corners of the double-pass images always showed a nonzero average intensity, even after background subtraction, but the calculated threshold level varied only in a small amount between subjects (average value: $12 \pm 5$ gray levels). In addition, we could not find any significant correlation between age and that residual intensity ( $r=-0.096, p=0.697$ ). This result means that the source of this stray light would be age independent, and thus probably linked to backscattering from the different optical surfaces of the experimental system and the eye (cornea and lens surfaces). 
As for the parameters of the scattering model, Fig. 7 shows the dependence of the equivalent diffuser roughness (i.e., standard deviation of $W_{\text {scat }}$ ) with age. The resulting average roughness for each age group is $0.038 \pm 0.018 \mu \mathrm{m}$ (30's), $0.049 \pm 0.015 \mu \mathrm{m}$ (40's), and $0.062 \pm 0.011 \mu \mathrm{m}$ (60's). Although we can observe a tendency toward a gradual increase of roughness with age, a linear regression (roughness versus age) provided a low Spearman's rank correlation coefficient ( $r=0.503, p=0.028$ ), due to the high spreading of the data, even within each age group. In fact, only the difference between the youngest and oldest groups was statistically significant. Therefore, concerning the amount of intraocular scattering, our measurements showed considerable variability among subjects, as well as an average trend toward an increase with age, which is consistent with previous findings. ${ }^{41}$

Figure 8 displays the dependence with age of the correlation length $r_{0}$ of the equivalent diffuser for all subjects. The resulting correlation length, averaged over all subjects, is $157 \pm 151 \mu \mathrm{m}$, showing a considerable amount of variability (almost 100\%) among subjects. This value is much higher than that expected from the size of possible ocular scatterers (about $10 \mu \mathrm{m}$ ). The group averages were $337 \pm 238 \mu \mathrm{m}$ (30's), $123 \pm 82 \mu \mathrm{m}$ (40's), and $88 \pm 30 \mu \mathrm{m}$ (60's), respectively, which suggests a progressive decrease of the correlation length with age, but again the correlation between both magnitudes is weak $(r=-0.457, p=0.049)$. Such values of the correlation length are associated to narrow scattering halos and hence a prevalence of forward, small-angle scattering over wide-angle scattering. Another way to understand these results is that in young eyes, with a high correlation length of $337 \mu \mathrm{m}$, what we are measuring is not scattering, but most probably we are measuring the effect of very high-order aberrations, not detected by the aberrometer. Nonetheless, there are a few eyes with much lower $r_{0}$ values, suggesting a clearer effect of scattering. In fact, the observed decrease of $r_{0}$ with age may mean a real increase of scattering. Nevertheless, as we discuss next, these results could be somewhat biased due to our measurement method, which has limitations to measure low intensity, wide-angle scattering halos.

\section{Discussion}

Our results so far support the presence of both very high-order aberrations and scattering, which cannot be detected by current aberrometers. For young eyes, very high-order aberrations with long correlation length of about $1 / 3 \mathrm{~mm}$ seems to explain the difference between aberrometric and double-pass MTFs, whereas we can observe an increase in forward, smallangle scattering with age. Nevertheless, the amount of intraocular scattering in normal young eyes seems close to the sensitivity of our experimental method, and hence relatively important systematic biases could be present.

First of all, double-pass measurements could be affected by backscattering from ocular surfaces other than the retina, especially from the anterior corneal surface in healthy eyes, or from the lens mostly in cataractous eyes. Although light coming from these layers is highly defocused on the CCD plane, its effect cannot be totally discarded. The impact of this even stray light on the modulation transfer function can be reduced by thresholding double-pass images, but this approach could potentially bias the MTF in eyes with increased forward scat-

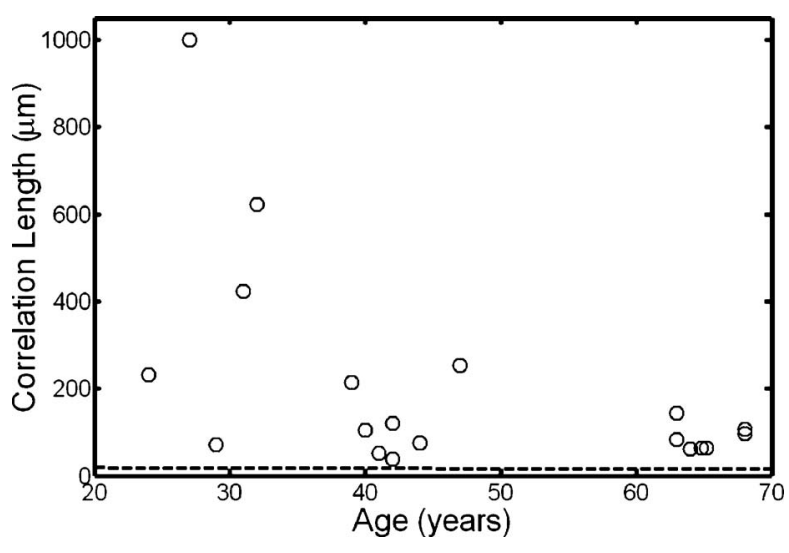

Fig. 8 Correlation length of the equivalent diffuser versus age. The dashed line marks the expected correlation length from the CIE small angle disability glare equation $(18 \mu \mathrm{m})$.

tering (corneal haze, cataracts, etc.). In normal eyes, retinal light levels at the visual fields corresponding to the image corners $(2.6 \mathrm{deg})$ are insignificant compared to the central maximum (below $10^{-4}$ ). However, in pathological eyes these corner levels could be significantly higher, due to an increased scattering halo, and thus thresholding could eliminate, to some degree, an important part of actual forward-scattered light unintentionally.

The difference between the aberrometric and double-pass MTFs was often masked by the measurement error. Experimental noise is a known issue in aberrometry, where modest signal-to-noise ratios (SNRs) of the order of 10 are common. ${ }^{42}$ In the present dual system, we found a slightly better performance of the double-pass device, which might be caused by the different number of samples taken by each subsystem in a single measurement ( 37 in the case of LRT versus 1 in the double-pass device).

In spite of this experimental variability, eight eyes still showed a statistically significant difference between devices, five of them corresponding to eyes previously identified as precataractous. Hence, there is not a complete correspondence between eyes with a statistically significant difference of MTFs and precataractous eyes. However, the diagnosis and grading of early stage cataracts is itself a highly subjective task for the clinician, involving a high level of uncertainty. In addition, the relation between ocular backscattering (estimated by means of the slit lamp) and forward scattering is not exactly known yet. Although a higher SNR would be desirable both in aberrometric and double-pass measurements, in the end it could be limited by the natural fluctuations and movements of the optical system of the eye.

The differences between aberrometric and double-pass MTFs were easily adjusted by a simple two-parameter phase screen equivalent diffuser model. The root-mean-square roughness $\sigma$ and the correlation length $r_{0}$ (mean grain size) are enough to fit the difference between both types of measurements. These two parameters determine the amount of scattering (roughness) and its spatial distribution (correlation length). The values of $\sigma$ and $r_{0}$ measured support a dim and compact scattering halo. In addition to the possible presence of very high-order aberrations, the long correlation lengths $(157 \pm 151 \mu \mathrm{m})$ obtained could be partly explained by poten- 
tial bias in the double-pass measurements. In particular, the limited field covered by the camera prevented us from recording scattering halos beyond $2 \mathrm{deg}$. For the measured correlation lengths $r_{0}$, more than $95 \%$ of the scattered intensity should be preserved within a 4 deg width patch. According to our calculations, however, this percentage should drop to $70 \%$ for $r_{0}=10 \mu \mathrm{m}$, and less than $10 \%$ for $r_{0}=1 \mu \mathrm{m}$. Hence, wide-angle scattering halos (low correlation lengths) would always be underestimated, whereas small-angle scattering halos would be favored by our measurement configuration. Conversely, a limited visual field would tend to overestimate the actual $r_{0}$. Additionally, in a first approximation, the peak intensity of the scattering halo is proportional $r_{0}^{2}$. This implies that, for a given roughness, the peak intensity in the halo quickly decreases with the correlation length. Then, the dynamic range of the camera could be insufficient to capture the whole double-pass aerial image, and again the system could be neglecting a potential source of scattering. These limitations (camera field of view and dynamic range) can hardly be avoided, since the spatial sampling is imposed by the Nyquist theorem in double-pass setups, whereas an increased dynamic range would have to struggle with backscattering noise in the end.

The equivalent diffuser model can be used to compare our results with a standard disability glare function. Despite that glare data are psychophysical and usually collected at higher angles than those involved here, there is a remarkable resemblance between the functional dependence with field angle between the scattering halo in our model (in the weak diffuser approximation) and that of the CIE small angle disability glare equation (SADGE). ${ }^{14}$ For the former,

$$
\begin{aligned}
\langle I(\xi, \eta)\rangle= & \frac{S^{2} n^{\prime 2}}{\lambda^{2} f^{\prime 2}} E \times \exp \left(-k^{2} \sigma^{2}\right) \\
& \times\left\{F_{0}(\xi, \eta)+\frac{2 \pi r_{0}^{2} k^{2} \sigma^{2}}{S}\left[1+\frac{k^{2} r_{0}^{2}\left(\xi^{2}+\eta^{2}\right)}{f^{\prime 2}}\right]^{3 / 2}\right\},
\end{aligned}
$$

where $\langle I(\xi, \eta)\rangle$ is the mean intensity distribution, $S$ is the pupil area, $n^{\prime}$ is the refractive index, $\lambda$ the wavelength, $f^{\prime}$ the focal length, $E$ the illuminance at the pupil plane, $F_{0}(\xi, \eta)$ the PSF without diffuser, $r_{0}$ the correlation length, $k$ the wave number, $\sigma$ the RMS roughness, and $(\xi, \eta)$ are spatial coordinates. Since $\left[\left(\xi^{2}+\eta^{2}\right) / f^{\prime 2}\right]^{1 / 2} \cong \theta$ for small angles, the angular behavior is quite similar to the CIE SADGE,

$$
\frac{L_{\text {veil }}}{E_{\text {glare }}}=\frac{10}{\theta^{3}}+\left[1+\left(\frac{\text { age }}{62.5}\right)^{4}\right] \times \frac{5}{\theta^{2}},
$$

where $L_{v e i l}$ is the equivalent veiling luminance $\left(\mathrm{cd} / \mathrm{m}^{2}\right)$, $E_{\text {glare }}$ is the glare illuminance on the eye (lux), $\theta$ is the glare angle (deg), and "age" is the age of the subject (years). Therefore, despite the different nature of the physical (intensity of the scattering halo) and psychophysical (disability glare function) magnitudes, both expressions correspond basically to the same function of $\theta^{-3}$ at small angles, thus allowing us a direct mathematical equality. In this way, it is possible to find the parameters $\sigma$ and $r_{0}$ of an equivalent diffuser, ${ }^{25}$ which would produce a scattering halo compatible with the CIE small angle disability glare equation. Thus, bearing in mind the different nature of the two functions that we are equating, we find that the equivalent diffuser for the CIE SADGE would have a root-mean-square roughness $\sigma=0.027 \mu \mathrm{m}$ and a correlation length $r_{0}=18 \mu \mathrm{m}$ (for a subject of 45 years). If we now compare with the average over all our subjects, the mean roughness is $0.051 \pm 0.017 \mu \mathrm{m}$, and only the youngest group gives a similar, but still higher, value $(0.038 \pm 0.018 \mu \mathrm{m})$. Therefore, the amount of scattering detected in our experiment is somewhat higher than that predicted by the CIE equation (dashed line in Fig. 7), in all subjects but two. Thus, the average roughness of the equivalent diffuser is almost double than that predicted from the CIE SADGE. There is an even larger discrepancy for the correlation lengths. While the disability glare equation would yield a value that is compatible with the possible scatterers (cells, fibers, etc.) in the eye, our results for young eyes, $r_{0}=337 \mu \mathrm{m}$, suggest the presence of very high-order aberrations rather than scattering.

Finally, we can compare our results with three similar recent studies on the effect of aging on ocular light scattering. ${ }^{17,26,27}$ Kuroda et al. ${ }^{17}$ used a single measurement with a Shack-Hartmann wavefront sensor $\left(\lambda_{m}=840 \mathrm{~nm}\right)$ to determine both aberrations and scattering. An empirical scattering index was used to estimate the amount of light scattered in each eye. Although this index cannot be easily related to our scattering parameters, it is interesting to note that they found a weak, though significant, correlation $(r=0.501, p$ $=0.001$ ) between age and scattering. In another experiment, more similar to ours, Shahidi and Yang $^{26}$ used a ShackHartmann wavefront sensor as well as a double-pass device to measure the line-spread function, both working at 543-nm wavelength. Basically, their light scattering index corresponds to the total amount of light scattered, which in our model can be expressed as the percentage $100 \times\left[1-\exp \left(-k^{2} \sigma^{2}\right)\right]$. They found scattering to increase by a factor of 1.4 between 39 and 69 years; our results support an increase by 1.75 in the same age range, which means a fairly good agreement. Nevertheless, the correlation between age and their light scattering index $(r=0.6, p=0.002)$ was superior to ours $(r=0.503, p$ $=0.028$ ), which could be due to differences between the eye populations examined (in particular, to their sparse sampling of old eyes). Finally, Díaz-Doutón et al. ${ }^{27}$ measured both aberrations and the double-pass aerial point-spread function $(\lambda$ $=780 \mathrm{~nm}$ )in several groups of subjects. Their results are summarized through a parameter consisting of a fraction of Strehl ratios. Such scattering parameters showed a considerable increase, a factor of 3 between 39 and 69 years, which is about double that found in similar experiments (1.4 in Shahidi and Yang; 1.75 present study). These differences can be explained by the fact that these studies differ either in the wavelength used, or in the scattering index or metric employed. Particularly, the chosen wavelength is determinant to minimize or not the contribution of scattering from deeper layers, and especially blood vessels from retina and choroid.

In spite of the known limitations in double-pass measurements, our experimental method provided a quick, objective assessment of ocular forward scattering; a magnitude more directly related to visual quality than backward scattering. The incorporation of scattering theory through the concept of 
equivalent diffuser allows a better quantitative analysis of the results. The characterization of the equivalent diffuser is rather compact, as it can be reduced conveniently to only two parameters. In this way, the overall optical quality of the eye could be reproduced by a set of Zernike coefficients (aberrations) plus two scattering parameters (roughness and correlation length). Our results suggest that the incorporation of an equivalent diffuser would be necessary not only for older but also for middle-aged subjects, whereas the optical quality of healthy, young eyes seems to be adequately described by the aberrometric measurements alone.

Some of the main approximations assumed here for normal eyes will not hold when the level of scattering increases, such as in cataractous eyes. In fact, the weak diffuser approximation could fail even for early stage cataracts. Furthermore, for more developed stages of cataracts, volume scattering models (rather than the rough surface, or phase screen, model used here) will be necessary. Nevertheless, this will be the subject of future work.

\section{Acknowledgments}

This work has been supported by the Comisión Interministerial de Ciencia y Tecnología (Spain), under grant DPI200204370-C02.

\section{References}

1. J. Liang, B. Grimm, S. Goelz, and J. F. Bille, "Objective measurement of wave aberrations of the human eye with the use of a Hartmann-Shack wave-front sensor," J. Opt. Soc. Am. A 11, 19491957 (1994).

2. R. Navarro and M. A. Losada, "Aberrations and relative efficiency of light pencils in the living human eye," Optom. Vision Sci. 74, 540 547 (1997)

3. G. Walsh, W. N. Charman, and H. C. Howland, "Objective technique for the determination of monochromatic aberrations of the human eye," J. Opt. Soc. Am. A 1, 987-992 (1984).

4. R. H. Webb, C. M. Penney, and K. P. Thompson, "Measurement of ocular local wavefront distortion with a spatially resolved refractometer," Appl. Opt. 31, 3678-3686 (1992).

5. J. Liang and D. R. Williams, "Aberrations and retinal image quality of the normal human eye," J. Opt. Soc. Am. A 14, 2873-2883 (1997).

6. M. B. Datiles, R. R. Ansari, and G. F. Reed, "A clinical study of the human lens with a dynamic light scattering device," Exp. Eye Res. 74, 93-102 (2002).

7. L. L. Holladay, "Action of a light source in the field of view in lowering visibility," J. Opt. Soc. Am. 14, 1-15 (1927).

8. Y. Le Grand, "Recherches sur la diffusion de la lumiére dans l'oeil humain," Rev. Opt., Theor. Instrum. 16, 240-266 (1937).

9. W. S. Stiles and B. H. Crawford, "The effect of a glaring light source on extrafoveal vision," Proc. R. Soc. London, Ser. B 122, 255-280 (1937).

10. G. A. Fry and M. Alpern, "The effect of a peripheral glare source upon the apparent brightness of an object," J. Opt. Soc. Am. 43, 189-195 (1953).

11. J. Walraven, "Spatial characteristics of chromatic induction: the segregation of lateral effects from stray light artifacts," Vision Res. 13, 1739-1753 (1973).

12. R. M. Boynton, J. M. Enoch, and W. R. Bush, "Physical measures of stray light in excised eyes," J. Opt. Soc. Am. 44, 879-886 (1954).

13. T. J. T. P. Van den Berg and J. K. Iljspeert, "Light scattering by donor lenses," Vision Res. 35, 169-177 (1995).

14. Commission Internationale de l'Éclairage, CIE. 146 CIE TC 1-50 Report CIE Equations for Disability Glare, CIE Collection on Glare, pp. 1-12 (2002).

15. G. Westheimer and J. Liang, "Evaluating diffusion of light in the eye by objective means," Invest. Ophthalmol. Visual Sci. 35, 2652-2657 (1994).

16. W. J. Donnelly, K. Pesudovs, J. D. Marsack, E. J. Sarver, and R. A.
Applegate, "Quantifying scatter in Shack-Hartmann images to evaluate nuclear cataract," J. Refract. Surg. 20, S515-S222 (2004).

17. T. Kuroda, T. Fujikado, S. Ninomiya, N. Maeda, Y. Hirohara, and T. Mihashi, "Effect of aging on ocular light scatter and higher order aberrations," J. Refract. Surg. 18, S598-S602 (2002).

18. J. M. Bueno, E. Berrio, M. Ozolinsh, and P. Artal, "Degree of polarization as an objective method of estimating scattering," J. Opt. Soc. Am. A 21, 1316-1321 (2004).

19. R. W. Hart and R. A. Farrell, "Light scattering in the cornea," J. Opt. Soc. Am. 59, 766-774 (1969)

20. G. B. Benedek, "Theory of transparency of the eye," Appl. Opt. 10, 459-473 (1971)

21. R. P. Hemenger, "Small-angle intraocular light scatter: a hypothesis concerning its source," J. Opt. Soc. Am. A 5, 577-582 (1988).

22. T. J. T. P. Van den Berg and H. Spekreijse, "Light scattering model for donor lenses as a function of depth," Vision Res. 39, 1437-1445 (1999).

23. B. Pierscionek, R. J. Green, and S. G. Dolgobrodov, "Intraocular light scattering as modelled through a stratified medium," Appl. Opt. 40, 6340-6348 (2001).

24. B. Pierscionek, R. J. Green, and S. G. Dolgobrodov, "Retinal images seen through a cataractous lens modelled as a phase-aberrating screen," J. Opt. Soc. Am. A 19, 1491-1500 (2002).

25. R. Navarro, "Incorporation of intraocular scattering in schematic eye models," J. Opt. Soc. Am. A 2, 1891-1894 (1985).

26. M. Shahidi and Y. R. Yang, "Measurements of ocular aberrations and light scatter in healthy subjects," Optom. Vision Sci. 81, 853-857 (2004)

27. F. Díaz-Doutón, A. Benito, J. Pujol, M. Arjona, J. L. Güell, and P. Artal, "Comparison of the retinal image quality with a HartmannShack wavefront sensor and a double-pass instrument," Invest. Ophthalmol. Visual Sci. 47, 1710-1716 (2006).

28. P. Artal, S. Marcos, R. Navarro, and D. R. Williams, "Odd aberrations and double-pass measurements of retinal image quality," J. Opt. Soc. Am. A 12, 195-201 (1995).

29. J. M. Gorrand, R. Alfieri, and J. Y. Boire, "Diffusion of the retinal layers of the living human eye," Vision Res. 24, 1097-1106 (1984).

30. N. López-Gil and P. Artal, "Comparison of double-pass estimates of the retinal-image quality obtained with green and near-infrared light," J. Opt. Soc. Am. A 14, 961-971 (1997).

31. J. K. Ijspeert, P. W. T. de Waard, T. J. T. P. van den Berg, and P. T. V. M. de Jong, "The intraocular straylight function in 129 healthy volunteers: dependence on angle, age and pigmentation," Vision Res. 30, 699-707 (1990).

32. G. M. Thurston, D. L. Hayden, P. Burrows, J. I. Clark, V. G. Taret, J. Kandel, M. Courogen, J. A. Peetermans, M. S. Bowen, D. Miller, K. M. Sullivan, R. Storb, H. Stern, and G. B. Benedek, "Quasielastic light scattering study of the living human lens as a function of age," Curr. Eye Res. 16, 197-207 (1997).

33. M. L. Hennelly, J. L. Barbur, D. F. Edgar, and E. G. Woodward, "The effect of age on the light scattering characteristics of the eye," Ophthalmic Physiol. Opt. 18, 197-203 (1998).

34. D. Sliney and M. Wolbarsht, Safety with Lasers and Other Optical Sources, Plenum, New York (1980).

35. M. Born and E. Wolf, Principles of Optics, Pergamon Press, Oxford (1983).

36. P. Rodriguez, R. Navarro, J. Arines, and S. Bara, "A new calibration set of phase plates for ocular aberrometers," J. Refract. Surg. 22, 275-284 (2006)

37. L. T. Chylack, J. K. Wolfe, D. M. Singer, M. C. Leske, M. A. Bullimore, I. L. Bailey, J. Friend, D. McCarthy, and S. Y. Wu, "The lens opacities classification system-III," Arch. Ophthalmol. (Chicago) 111, 831-836 (1993)

38. P. Beckmann, "Scattering of light by rough surfaces," Prog. Opt. 6 , 55-69 (1967).

39. P. Beckmann, "Scattering by composite rough surfaces," Proc. IEEE 53, 1012-1015 (1965).

40. P. Artal, M. Ferro, I. Miranda, and R. Navarro, "Effects of aging in retinal image quality," J. Opt. Soc. Am. A 10, 1656-1662 (1993).

41. J. J. Vos, "Disability glare—a state of the art report," CIE J. 3, 39-53 (1984).

42. P. Rodríguez, R. Navarro, L. González, and J. L. Hernández, “Accuracy and reproducibility of Zywave, Tracey and experimental aberrometers," J. Refract. Surg. 20, 810-817 (2004). 